\title{
Topology Management for Mobile Ad Hoc Networks Scenario
}

\author{
Ashish Mandloi ${ }^{1}$, Girish Kaushal ${ }^{2}$ \\ ${ }^{1,2}$ Lecturer, Shri Vaishanv Polytechnic, Indore (MP)
}

\begin{abstract}
Cooperative communication is the main accessing point in present days. These results can be accessed through proactive protocol like route request packet sending and route request packet receiving. The main issue is how communication will be done in MANETS. Mobile Ad-hoc networks are self-configurable networks; each node behaves like server and client in MANET. COCO (Capacity Optimized Cooperative Communication) model was developed for accessing these types of resources in MANETs. This model can't provide sufficient communication or overall network performance. This model provides sufficient capability improvement in mobile ad-hoc networks, but this model will be taking more power resources for doing this work. exploitation simulation examples, we have a tendency to show that physical layer cooperative communications have important impacts on the performance of topology control and network capability, and also the proposed topology management scheme will considerably improve the network capability in MANETs with cooperative communications.
\end{abstract}

Keyword- Topology Control, Cooperative Communication, MANET, Network Capacity.

\section{INTRODUCTION}

The demand for speed in wireless networks is incessantly increasing. Recently, cooperative wireless communication has received tremendous interests as an untapped suggests that that for improving the performance of information transmission operative over the ever-challenging wireless medium. Cooperative communication has emerged as a replacement dimension of diversity to emulate the methods designed for multiple antenna systems, since a wireless mobile device might not be able to support multiple transmit antennas due to size, value, or hardware limitations [1], [2]. By exploiting the broadcast nature of the wireless channel, cooperative communication permits single-antenna radios to share their antennas to make a virtual antenna array, and offers important performance enhancements. This promising technique has been thought of within the IEEE 802.16j normal, and is predicted to be integrated into 3GPP-LTE multi-hop cellular networks [3].

Although some works are done on cooperative communications, most existing works are focused on link-level physical layer issues, like outage chance and outage capability [4]. Consequently, the impacts of cooperative communications on network-level higher layer problems, like topology management, routing and network capability, are largely ignored. Indeed, most of current works on wireless networks decide to produce, adapt, and manage a network on a maze of point-to-point non-cooperative wireless links. Such architectures is seen as advanced networks of easy links. However, recent advances in cooperative communications can give variety of advantages in flexibility over traditional techniques. Cooperation alleviates certain networking problems, like collision resolution and routing, and permits for less complicated networks of further advanced links, instead of difficult networks of straightforward links.

Therefore, several higher layer aspects of cooperative communications advantage further analysis, e.g., the impacts on topology management and network capability, particularly in mobile ad-hoc networks (MANETs), which may establish a dynamic network whereas not a collection infrastructure. A node in MANETs will operate every as a network router for routing packets from the Different nodes and as a network host for transmission and receiving information. MANETs are notably useful once a reliable mounted or mobile infrastructure is not available. Instant conferences between notebook computer users, military applications, emergency operations, and alternative secure-sensitive operations are necessary applications of MANETs because of their quick and easy preparation.

Due to the shortage of centralized management, MANETs nodes get together with each other to realize a typical goal. the main activities concerned in organization are neighbour discovery, topology organization, and topology reorganization. topology describes the property data of the complete network, together with the nodes among the network and conjointly the connections between them. Topology management is incredibly necessary for the general performance of a painter. for instance, to take care of a reliable network property, nodes in MANETs may match at the most radio power, which ends in high nodal degree and long link distance, however further interference is introduced into the 
network and so much less turnout per node is obtained. Exploitation topology management, a node rigorously selects a collection of its neighbours to see logical information links and dynamically modification its transmit power consequently, thus on succeed high throughput among the network whereas keeping the energy consumption low [5].

In this paper, considering each higher layer network capability and physical layer cooperative communications, we tend to study the topology control issues in MANETs with cooperative communications. we tend to propose a CapacityOptimized Cooperative (COCO) topology management theme to enhance the network capability in MANETs by put together optimizing transmission mode alternative, relay node choice, and interference management in MANETs with cooperative communications. exploitation simulation examples, we tend to show that physical layer cooperative communications have very important impacts on the performance of topology management and network capability, and also the projected topology management theme can significantly improve the network capability in MANETs with cooperative communications.

\section{MOBILE AD HOC NETWORKS WITH TOPOLOGY CONTROL}

The constellation during a painter is changing dynamically owing to user quality, traffic, node batteries, etc. Meanwhile, the topology during a painter is manageable by adjusting some parameters like the transmission power, channel assignment, etc. In general, topology control is such a theme to work out wherever to deploy the links and also the approach the links add wireless networks to create an honest constellation, which will optimize the energy consumption, the capability of the network, or end-toend routing performance. Topology management is originally developed for wireless detector networks (WSNs), Mobile Ad-hoc Networks, and wireless mesh networks to cut back energy consumption and interference. it always leads to a easier constellation with very little node degree and short transmission radius, which will have high-quality links and fewer competition in medium access management (MAC) layer. Spatial/spectrum reprocess will become potential owing to the smaller radio coverage. alternative properties like symmetry and planarity are expected to get inside the resultant topology. Symmetry can facilitate wireless communication and two-way acknowledgment schemes for link acknowledgment whereas planarity will increase the probability for parallel transmissions and house reprocess.

Power management and channel management problems are let alone topology management in MANETs whereas they are treated one by one traditionally. though a mobile node can sense the available channel, it lacks of the scope to create network-wide alternatives. It thus makes further sense to conduct power management and channel management via the topological viewpoint. The goal of topology management is then to line up interference-free connections to minimizes the most transmission power and also the range of required channels. it's conjointly fascinating to construct a reliable constellation since it will end in some benefits for the network performance.

\section{TOPOLOGY CONTROL FOR NETWORK CAPABILITY IMPROVEMENT IN MANETS WITH COOPERATIVE COMMUNICATIONS}

In this section, we have a tendency to initial describe the capability of MANETs. Then, we have a tendency to present the projected Capacity-Optimized COoperative (COCO) topology management scheme for MANETs with cooperative communica-tions.

A. The capability of MANETs

As a key indicator for the knowledge delivery ability, network capability has attracted tremendous interests since the landmark paper by Gupta and Kumar [8]. There are completely different definitions for network capability. 2 styles of network capability are introduced in [8]. the primary one is transport capability, that is analogous to the whole one-hop capability within the network. It takes distance into thought and is predicated on the total of bit-meter product. One bit-meter means one bit has been transported to a distance of 1 meter toward its destination. Another kind of capability is turnout capability, that is predicated on the knowledge capability of a channel. Obviously, it's the quantity of all the information with success transmitted throughout a unit time. it's been shown that the capability in wireless circumstantial networks is restricted. In ancient MANETs while not cooperative communications, the capability is remittent because the range of nodes within the network will increase. Asymptotically, the per-node turnout declines to zero once the quantity of nodes approaches to eternity [8]. during this study, we have a tendency to adopt the second kind of definition.

The expected network capability is decided by varied factors: wireless channel rate within the physical layer, abstraction reprocess planning and interference within the link layer, topology management conferred in segment II.B, traffic balance in routing, traffic patterns, etc. within the physical layer, channel rate is one amongst the most factors. in theory, \{channel capability|data rate $\}$ is derived exploitation technologist capacity formula. In apply, wireless channel rate is put together determined by the modulation, channel writing, transmission power, fading, etc. additionally, outage capability is typically employed in apply, that is 
supported by alittle outage chance, to represent the link capability.

In the link layer, the abstraction reprocess is that the major ingredient that affects network capability. Link interfer-ence, that refers to the affected nodes throughout the transmission, conjointly features a vital impact on network capability. Higher interference could scale back coincidental transmissions within the network, therefore scale back the network capability, and the other way around. The mackintosh operate ought to avoid collision with existing transmission. It uses a abstraction and temporal planning in order that coincidental transmissions don't interfere with one another. Nodes at intervals the transmission vary of the sender should keep silent to avoid destroying ongoing transmissions. additionally, there are some factors that forestall the data rate from being totally utilised, like hidden and exposed terminals, which require to be solved exploitation hand-shake protocols or a fanatical management channel in wireless networks.

Routing not solely finds ways to satisfy quality of service (QoS) necessities, however conjointly balances traffic hundreds in nodes to avoid hot spots within the network. By leveling traffic, the network could admit additional traffic flows and maximize the capability. Since we have a tendency to specialise in topology management and cooperative communications, we have a tendency to assume a perfect load balance within the network, wherever the traffic hundreds within the network are uniformly distributed to the nodes within the network.

The study in [4] shows that cooperative transmissions don't continuously crush direct transmissions. If there exists no such a relay that creates cooperative transmissions have larger outage capability, we have a tendency to rather transmit data directly or via multi-hops. For this reason, we want to work out the most effective link block (see Fig. 1) and also the best relay to optimize link capability. On the opposite hand, alternative nodes in

The transmission vary ought to be silent so as to not disrupt the transmission owing to the open shared wireless media. The affected areas embody the coverage of the supply, the coverage of the destination, similarly because the coverage of the relay.

B. Rising Network Capability Exploitation Topology Management in MANETs With Cooperative Communications

To improve the network capability in MANETs with cooperative communications exploitation topology management, we will set the network capability because the objective operate within the topology management downside. so as to derive the the network capability during a painter with cooperative communications, we want to get the link capability and abstract thought model once a selected transmission manner (i.e., direct transmission, multi-hop transmission, or cooperative transmission) is employed.

When ancient transmission mechanism is employed, given alittle outage chance, the outage link capability is simply derived [9]. Since solely 2 nodes are concerned within the transmission mechanism, the interference set of a right away transmission is that the union of coverage sets of the supply node and also the destination node. during this paper, we have a tendency to adopt the interference model in [8], that ambit synchronal transmissions within the section of the transmitter and receiver. This model fits the medium access control operation well, e.g., the popular IEEE $802.11 \mathrm{mac}$ in most mobile devices in MANETs. Herein, interference of a link is outlined as some combination of coverage of nodes concerned within the transmission.

Multi-hop transmission is illustrated exploitation two-hop transmission. once two-hop transmission is employed, 2 time slots are consumed. within the initial slot, messages are transmitted from the supply to the relay, and also the messages are forwarded to the destination within the second slot. The outage capability of this two-hop transmission is derived considering the outage of every hop transmission. The transmission of every hop has its own interference, that happens in several slots. Since the transmissions of the 2 hops cannot occur at the same time however in two separate time slots, the end-to-end interference set of the multi-hop link is decided by the most of the 2 interference sets.

When cooperative transmission is employed, a best relay has to be designated proactively before trans-mission. during this study, we have a tendency to adopt the decode-and-forward relaying theme. The supply broadcasts its messages to the relay and destination within the initial slot. The relay node decodes and re-encodes the signal from the supply, then forwards it to the destination within the second slot. The 2 signals of the supply and also the relay are decoded by highest rate combining at the destination. the most instant end-to-end mutual data, outage chance, and outage capability is derived [4]. For the interference model, within the broadcast amount, each the lined neighbors of the supply and also the lined neighbors of the relay and also the destination ought to be silent to make sure no-hit receptions. within the second slot, each the lined neighbors of the chosen relay and also the destination ought to be silent to make sure no-hit receptions.

After getting the link capability and abstract thought models, the network capability is derived [7] because the objective operate within the topology management downside (1). By considering transmission mechanism, multi-hop transmission, cooperative transmission, and interference, the 
projected palm tree topology management theme extends physical layer cooperative communications from the link-level perspective to the network-level perspective in MANETs. The projected theme will confirm the most effective kind of transmission and also the best relay to optimize network capability.

Two constraint conditions got to be taken into thought within the projected coco topology control theme. One is network property, that is that the basic demand in topology control. The end-to-end network property is secured via a hop-by-hop manner within the objective operate. each node is guilty of the connections to any or all its neighbors. If all the neighbor connections are secured, the end-to-end property within the whole network is preserved. the opposite facet that determines network capability is that the path length. Associate in Nursing end-to-end transmission that traverses additional hops can import additional information packets into the network. though path length is especially determined by routing, coco limits dividing an extended link into too several hops domestically. The limitation is 2 hops owing to the very fact that solely two-hop relaying is adopted.

\section{CONCLUSIONS AND FUTURE WORK}

In this work, we have introduced physical layer cooperative communications, topology control and network capability in MANETs. To improve the network capability of MANETs with cooperative communications, we've projected a CapacityOptimized Cooperative (COCO) topology management scheme that considers every higher layer network capability and physical layer relay alternative in cooperative communications. Simulation results have shown that physical layer cooperative communications model have vital impacts on the performance of topology management and network capability, and also the projected topology management scheme can significantly improve the network capability in MANETs with cooperative communications. Future work is ongoing to contemplate dynamic traffic patterns among the projected scheme to further improve the performance of MANETs with cooperative communications.

\section{REFERENCES}

[1]. J. Laneman, D. Tse, and G. Wornell, "Cooperative diversity in wireless networks: Efficient protocols and outage behavior," IEEE Trans. Inform. Theory, vol. 50, no. 12, pp. 3062-3080, 2004.

[2]. V. Mahinthan, L. Cai, J. Mark, and X. Shen, "Partner selection based on optimal power allocation in cooperative-diversity systems," IEEE Trans. Veh. Tech., vol. 57, pp. $511-$ 520, Jan. 2008.

[3]. P. H. J. Chong, F. Adachi, S. Hamalainen, and V. Leung, "Technologies in multihop cellular network," IEEE Commun. Magazine, vol. 45, pp. 64-65, Sept. 2007.

[4]. K. Woradit, T. Quek, W. Suwansantisuk, M. Win, L. Wuttisittikulkij, and H. Wymeersch, "Outage behavior of selective relaying schemes," IEEE Trans. Wireless Commun., vol. 8, no. 8, pp. 3890-3895, 2009.

[5]. P. Santi, "Topology control in wireless ad hoc and sensor networks," ACM Computing Surveys (CSUR), vol. 37, no. 2, pp. 164-194, 2005.

[6]. T. Cover and A. E. Gamal, "Capacity theorems for the relay channel," IEEE Trans. Inform. Theory, vol. 25, pp. 572-584, Sept. 1979.

[7]. Q. Guan, S. Jiang, Q. Ding, and G. Wei, "Impact of topology control on capacity of wireless ad hoc networks," in Proc. IEEE ICCS, (Guangzhou, P.R. China), Nov. 2008.

[8]. P. Gupta and P. Kumar, "The capacity of wireless networks," IEEE Trans. Inform. Theory, vol. 46, no. 2, pp. 388-404, 2000.

[9]. A. Goldsmith, Wireless Communications. Cambridge University Press, 2005.

[10]. M. Burkhart, P. von Rickenbach, R. Wattenhofer, and A. Zollinger, "Does topology control reduce interference?," in Proc. 5th ACM Int. 